\title{
Características Personales y Práctica Docente de Profesores Universitarios y su Relación con la Evaluación del Desempeño
}

\author{
Personal Characteristics and Teaching Practice of University \\ Professors and their Relation with the Performance Evaluation
}

\author{
José Osvaldo Galván Salinas \\ Gabriela María Farías Martínez * \\ Tecnológico de Monterrey, México
}

\begin{abstract}
Este artículo presenta los resultados de una investigación realizada en una institución de educación superior, con el propósito de conocer las características de un profesor inspirador y aquellos factores y prácticas docentes que resultan más significativos para los alumnos cuando evalúan a sus profesores, a fin de generar estrategias para mejorar el desempeño docente. Se utilizaron métodos de investigación mixtos (cuantitativo y cualitativo). Se aplicaron entrevistas y cuestionarios a profesores, estudiantes y administrativos; también se hicieron observaciones de actividades y reuniones. Los factores analizados fueron la práctica docente, la formación académica, experiencia en el ámbito profesional, edad, género y otros. Los resultados indicaron que existe relación entre las características personales y de práctica docente con los resultados de la evaluación del desempeño de los profesores. Se logró, además, identificar las características y prácticas de mayor significancia para esta relación. Se concluyó, asimismo, que las generaciones actuales son exigentes con el desempeño de sus profesores y cuestionan el porqué de cada actividad y la utilidad de la misma para su futuro profesional.
\end{abstract}

Palabras clave: Evaluación de profesores; Desempeño del profesor; Educación superior; Evaluación del desempeño; Práctica docente; Profesores universitarios.

This article presents the results of an investigation carried out in a higher education institution, with the objective of finding the characteristics of an inspiring teacher and those factors and teaching practices that are more significant for the students when they evaluate their professors, and thus, generate strategies to improve teaching performance. Mixed research methods were used (quantitative and qualitative). Interviews and questionnaires were applied to professors, students and administrators; observations of activities and meetings were also made. The analyzed factors on the professors were the teaching practice, the academic formation, experience in the professional field, age, gender and others. The results indicated that there is a relationship between personal characteristics and teaching practice with the results of the evaluation of teachers' performance. It was also possible to identify the most significant characteristics and practices for this relationship. Also, it was concluded, that current generations are demanding with the performance of their professors and question the reason of each activity and the utility of it for their professional future.

Keywords: Teacher evaluation; Student evaluation of teacher performance; Higher education; Performance evaluation; Teaching practice; University professors.

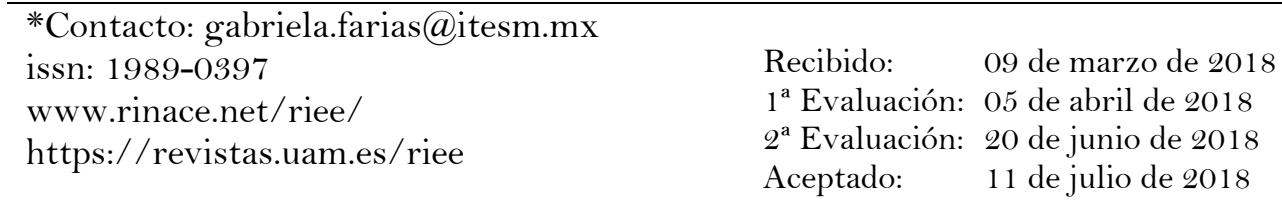




\section{Antecedentes del problema de investigación}

La evaluación docente es una práctica que no necesariamente se utiliza para retroalimentar de manera eficiente a los profesores y ayudarlos a mejorar su labor. Un aporte importante a esta idea es que las pruebas estandarizadas no captan todas las cualidades de un proceso de enseñanza-aprendizaje exitoso (Duncan, 2011), es por esto que siempre se requieren medidas múltiples en la evaluación de maestros, escuelas, distritos y estados.

A continuación, se presenta la revisión de literatura relacionada con el problema de investigación.

\section{Revisión de Literatura}

\subsection{Práctica docente}

Puede entenderse como práctica docente (Achilli, 1988) el trabajo que el maestro desarrolla cotidianamente en determinadas y concretas condiciones sociales, históricas e institucionales, adquiriendo una significación tanto para la sociedad como para el propio maestro. El proceso se desarrolla en el contexto del aula, mismo que pone de manifiesto una determinada relación maestro-conocimiento-alumno, centrada en el "enseñar" y el "aprender".

Fierro, Fortoul y Rosas (1999) proponen otra aproximación al estudio de la práctica docente donde presentan seis dimensiones enlistadas y explicadas a continuación:

a) Dimensión personal: se refiere a la actividad personal realizada por un ser humano. Implica que cada persona es única, con cualidades, características y dificultades que son resultado de vivencias. Un ser en constante evolución con ideales, motivos, proyectos y circunstancias que, de manera natural, se despliegan en la vida profesional.

b) Dimensión institucional: la docencia se desarrolla dentro de una organización con características y regulaciones propias en la cual el maestro, con su trabajo, aporta colectivamente. Es decir, en esta dimensión se reconoce que el actuar profesional de cada maestro está influenciado por su experiencia y pertenencia en la institución.

c) Dimensión interpersonal: esta se enfoca en las relaciones que establece el maestro con las personas que participan en el proceso educativo, es decir, alumnos, compañeros de trabajo, directores y padres de familia. Existen diferencias entre estos individuos, tales como edad, género, escolaridad, intereses, ideologías, preferencias políticas y creencias religiosas; todas estas influyen en el proceso de enseñanza.

d) Dimensión social: la enseñanza se da en un entorno histórico, político, social, geográfico, cultural, económico, industrial y tecnológico muy particular, en el que el resultado de esta práctica influye directamente. Esta dimensión pretende analizar y observar cómo reacciona el profesor a su entorno y contex to social.

e) Dimensión didáctica: se refiere a la función de enseñar y dirigir al maestro para que sus alumnos puedan adquirir el saber colectivo culturalmente organizado y 
puedan construir su propio conocimiento. Evalúa la naturaleza y la profundidad con la que los alumnos pueden recrear aprendizajes significativos.

f) Dimensión sobre los valores: la práctica docente no se presenta de manera neutral, conlleva intrínsecamente una referencia axiológica, es decir, un conjunto de valores. Cada maestro transfiere, de forma continua consciente o inconsciente, sus propios valores, su forma de entender el mundo, de valorar las relaciones y humanas y de apreciar el conocimiento.

La suma de estas dimensiones influencia fuertemente en cómo los alumnos forman sus ideas, actitudes y modos de interpretar la realidad. Se vuelven elementos que deben ser considerados en la evaluación del desempeño docente.

\subsection{Evaluación del desempeño docente}

Uno de los fundamentos en las teorías administrativas, de gestión y de sistemas de calidad es realizar evaluaciones para poder medir, conocer y mejorar. De igual forma, para poder mejorar, el ámbito educativo debe ser evaluado en sus distintos elementos: investigación, divulgación, administración de instituciones, diseño de programas de estudio, procesos de enseñanza-aprendizaje, instalaciones, plataformas tecnológicas, recursos pedagógicos o andragógicos y, de los más importantes, el desempeño docente (Muñoz Cantero, Rios de Deus y Abalde, 2002). Enfocados en definir la "Evaluación del desempeño docente" en el contexto educativo, Tejedor y García-Valcárcel (2010), afirman que una evaluación es un proceso complejo y cognitivo, ya que en él se construyen conocimientos; instrumental, porque requiere del diseño y aplicación de determinados procedimientos y métodos; y axiológico, debido a que supone siempre establecer el valor de algo. De manera similar, Rueda, Schmelkes y Díaz-Barriga (2014) definen a la "Evaluación del desempeño docente" como el proceso de análisis estructurado, reflexivo y autorreflexivo, que permite conocer la naturaleza del objeto de estudio y emitir juicios de valor del mismo, proporcionando información para ayudar a mejorar y ajustar la acción educativa. Su finalidad, dice, es retroalimentar. Uno de sus propósitos es facilitar y promover el cambio (Arbesú y Rueda, 2003). Por otra parte, desempeño significa cumplir con una responsabilidad del mejor modo posible e involucrando de manera interrelacionada los saberes, habilidades, actitudes, valores y competencias de cada uno, buscando siempre la mejora y el logro de niveles de actuación cada vez más elevados.

Los antecedentes muestran que, al limitarse a la comprobación de logros académicos o administrativos obtenidos, las evaluaciones eran sesgadas y poco objetivas (Castillo y Cabrerizo, 2010). Actualmente, la evaluación se ha enfocado más a procedimientos de asesoramiento, de regulación, de reorientación y ordenación de los aprendizajes para la mejora del proceso de enseñanza-aprendizaje.

Los profesores Lukas y Santiago (2014) realizan una clasificación de los antecedentes de la evaluación educativa:

- Antecedentes remotos (2000 a. C.-1900)

- Etapa de la eficiencia y de los test (1901-1929)

- Etapa de Tyler (1930-1945)

- Etapa de la inocencia (1946-1957)

- Etapa del realismo o de expansión (1958-1972) 
- Etapa de la profesionalización (1973-a la fecha)

Hay evidencia de que China aplicaba exámenes a los candidatos a ingresar a la administración del estado. Por otro lado, Sócrates utilizaba cuestionarios como parte de su metodología didáctica. También, se han hallado escritos jesuitas de 1599 que servían como guías para estudiar para exámenes escritos. Por otro lado, en 1845 se llevó a cabo un estudio en Boston con el fin de comprobar si las escuelas de esa región daban una educación de calidad a sus alumnos, este acto demuestra la inquietud que existe por construir bases para buscar la mejora educativa, incluyendo la evaluación docente.

La Etapa de la inocencia se vio caracterizada por aportar a la utilización del test: la clasificación y el ordenamiento de los objetivos educativos. A inicios del siglo XX comenzó a haber una investigación educativa más formal con estudios empíricos y técnicas estadísticas. El Círculo de Viena y la Escuela de Chicago hicieron importantes aportes, el primero con el neopositivismo y la segunda con la metodología del enfoque cualitativo. Añadido a esto, Tyler (citado en Angulo y Blanco, 1994) aportó los conceptos de currículum y de evaluación.

La Etapa de expansión se vio favorecida por el gobierno de Estados Unidos, quien destinó fondos federales a proyectos encaminados a lograr metas educativas. Además, mejoró programas curriculares y exigió evaluaciones a profesores y directivos.

Por último, durante la Etapa de profesionalización, la evaluación educativa se vuelve una rama individual del ámbito educativo al separarse de la investigación educativa (García y Vaillant, 2010). Surgen cursos y revistas especializadas en la evaluación educativa.

Actualmente, la técnica de evaluación educativa más utilizada en Norteamérica, Europa y Asia es por medio de cuestionarios de evaluación del docente respondidos por los alumnos (Rueda, 2008). Sin embargo, esta técnica ha creado debate (Theall y Franklin, 2000), pues se considera que hay un uso excesivo de cuestionarios; por otra parte, también se piensa que estos datos pueden resultar útiles para investigaciones más detalladas como la presente. Un problema detectado por estos mismos autores es que la evaluación educativa se ha orientado más a buscar métodos de medición que a buscar la mejora (Theall y Franklin, 2000). Esta revisión de antecedentes muestra que la evaluación educativa es una estrategia importante y en desarrollo, que tiene gran importancia en la toma de decisiones en políticas educativas (Elizalde y Reyes, 2008).

Es importante considerar que la evaluación académica no se reduce a sólo la práctica de transmisión de conocimientos, sino también a cómo los docentes contribuyen a la formación intelectual de los alumnos, la riqueza de los contenidos, las estrategias cognitivas empleadas, la asimilación de competencias y la educación en valores y actitudes para la formación de profesionistas que aporten de manera positiva a nuestra sociedad. Es por esto que Rueda y Díaz-Barriga (2011) consideran que las evaluaciones a profesores se deben realizar en torno a dos aspectos: la parte académica y la docente. Refiriéndose la primera a la impartición de clases, asesorías, tutoría, difusión de cultura, elaboración de materiales didácticos y de investigación. Mientras que la segunda, se enfoca directamente a lo relacionado con el proceso de enseñanza-aprendizaje, a su actividad cognitiva. En cuanto al objetivo de las evaluaciones académicas, Andrews y Barnes (1990, citado en Rueda y Díaz, 2011), indican que existen dos posiciones respecto a su función: formativa y sumativa. La primera se centra en los procesos e intenta aportar información sobre las 
actividades propias de la función y desempeño del profesor, en busca de su mejora. La segunda está enfocada hacia la valoración del producto o del desempeño docente, siendo esencial asignarle una calificación, como un indicador de la efectividad de su labor.

Un caso de evaluación docente más reciente es el cuestionario de opinión del estudiante utilizado dentro de la Facultad de Medicina de la UNAM. En este caso, el cuestionario fue aplicado a estudiantes que cursaron el $1^{\circ} \mathrm{o} 2^{\circ}$ año de la carrera durante el año académico de 2007 a 2008. El estudio recogió 20,136 cuestionarios, cada uno compuesto por 36 reactivos para los cursos de teoría y de 41 para los cursos de prácticas. Estos evaluaban distintos rubros como: Puntualidad y asistencia, Cumplimiento con el programa del curso, Metodología, Materiales de apoyo, Actitud, Evaluación de los aprendizajes, Satisfacción y Actividades prácticas. De la implementación de dichos cuestionarios en la Facultad de Medicina, se concluyó que estos "pueden emplearse para elaborar diferentes comparaciones y reportes institucionales evaluativos sobre el estado que, desde la perspectiva del estudiante -su destinatario principal-guardan las actividades de docencia" (Martínez González, Sánchez Mendiola y Martínez Stack, 2010).

Una investigación similar fue la realizada por Vásquez-Rizo y Gabalán-Coello (2012) en una universidad pública ubicada en el suroccidente colombiano. Fue un estudio cuantitativo con análisis multivariado. Para la investigación se recopiló información de la percepción estudiantil por medio de un cuestionario de opinión de alumnos. Sin embargo, en el estudio colombiano se ingresaron y procesaron las percepciones del jefe inmediato y la autoevaluación de cada maestro. De los resultados arrojados por este estudio se pudo percibir cuáles son los factores que más se toman en cuenta dentro de la comunidad estudiantil y de profesores de la escuela estudiada, al momento de hacer evaluaciones. Además, como se mencionó, también profesores y jefe inmediato realizaron autoevaluaciones, resultando en calificaciones que oscilaban entre "Bueno" y "excelente". Esto da pie a futuras investigaciones para resolver si estos resultados se deben a que realmente hay altos estándares de calidad o si los profesores responden de forma subjetiva (Vásquez-Rizo y Gabalán-Coello, 2012).

\subsection{Prácticas e instrumentos para la evaluación del desempeño docente}

Instrumento es definido por Rueda y Díaz-Barriga (2011) como aquellos objetos o dispositivos específicos que se utilizan para medir, regular o analizar algún proceso. En el caso de la evaluación docente, un instrumento puede ser una prueba, cuestionario, escala de opinión, diario de observación en el aula o entrevistas (Díaz-Barriga y Rueda, 2000).

Hasta el presente, no existe un estándar en metodologías o instrumentos a nivel mundial que midan o evalúen las prácticas educativas. Martínez (2013) comparó dos sistemas educativos de clase mundial, el de Singapur y el de Estados Unidos de América. Observó que el de Singapur se ha caracterizado por obtener importantes reconocimientos educativos y por tener altos estándares de evaluación por maestro. Este sistema se ha vuelto referente como caso de estudio por sus prácticas educativas vanguardistas. De manera similar, el sistema estadounidense muestra grandes avances, de hecho, se posiciona como el país con el mayor número de universidades mejor calificadas a nivel mundial (Guadagni, 2015). A pesar de que ambos son ejemplos de buenos sistemas educativos, presentan enfoques distintos; en Singapur, la práctica educativa está orientada a aspectos afectivos y de desarrollo de la autoconfianza de los alumnos; por otro lado, la 
estrategia de Estados Unidos está más enfocada a aspectos técnicos como la claridad de la enseñanza y planificación de clases.

Castillo (2010) plantea la utilización de la observación, la entrevista, los test, las encuestas, las escalas, las fichas, entre otros. Permitiendo así, valorar en cada momento la calidad, el grado de desempeño y las competencias adquiridas y desplegadas para la labor docente. Luna y Torquemada (2008), sostienen que las dos medidas de confiabilidad más utilizadas en la evaluación docente son la consistencia de la medida y la estabilidad de los puntajes a través del tiempo. Estos autores proponen que la validez de los puntajes otorgados por los estudiantes al profesor se estudie desde dos perspectivas: como una medida que muestra la opinión de los estudiantes acerca de la calidad de la instrucción y otra como una forma adecuada de reflejar la efectividad de la enseñanza.

A través de estudios realizados por él mismo, Páramo (2008) ha concluido que las evaluaciones realizadas por los alumnos hacia los docentes, no deben ser la única herramienta utilizada para evaluar a los profesores. Factores tales como el género, la personalidad, el tipo de trato, entre otros, pueden afectar y sesgar la evaluación que un alumno haga de su profesor, es por esto que concluye que no debe ser el único instrumento. Martínez (2013) desarrolló estudios que le permitieron notar que, ya sea en vivo o en grabación, la observación del docente es una herramienta útil.

Martínez (2013) también habla sobre el propósito y el uso que se da a los resultados de las evaluaciones hechas a los profesores. Estos van desde brindar incentivos a los maestros mejor evaluados, proveer de información para las políticas educativas e identificar las prácticas eficaces con el fin de encontrar modelos de enseñanza innovadores que puedan ser replicados en todo el sistema. Añadido a esto, las evaluaciones también permiten identificar a los maestros con ciertas problemáticas que pueden ser actitudinales, de capacitación, limitaciones de competencias, y otras.

Tejedor y García-Valcárcel (2010) hablan de una doble función de las evaluaciones docentes. La primera, siendo instrumento técnico de control a modo de diagnóstico, incorpora una perspectiva de análisis que prevé ciertas hipótesis explicativas que facilitarán la toma de decisiones. La segunda supone concebir a los docentes como actores sociales de cambio, que reciben los resultados de sus evaluaciones como oportunidades de mejora personal y académica.

\section{Método}

Realizar trabajo de investigación en el tema de educación demanda comprensión en muy diversas dimensiones. Shulman (1986, citado por Pereira Pérez, 2011) plantea que:

El conocimiento no crece de forma natural e inexorable. Crece por las investigaciones de los estudiosos (empíricos, teóricos, prácticos) y es por tanto una función de los tipos de preguntas formuladas, problemas planteados y cuestiones estructuradas por aquellos que investigan. (pp. 9-10)

El método o enfoque metodológico que se empleó en esta investigación fue el mixto. La razón para elegir este enfoque es que permite obtener y potenciar las ventajas de cada paradigma, el cualitativo y el cuantitativo (Alvarado, Canales y Pineda, 1994). Esto, con la idea de disminuir la probabilidad de dejar fuera elementos críticos que pudieran aportar a la investigación. 
Pérez (2011) señala que a partir de los años 90’s, los métodos de investigación mixtos cobraron auge y se hicieron muy útiles en campos como la educación, la medicina, la enfermería, la comunicación y la psicología. Con su tesis demuestra que el uso de más de un enfoque de investigación potencia la posibilidad de comprensión de los fenómenos en estudio, especialmente si estos se refieren a campos complejos en donde está involucrado el ser humano y su diversidad, como es nuestro caso de estudio.

Johnson y Onwuegbuzie (2004) se refieren a los diseños mixtos como el tipo de estudio donde el investigador mezcla o combina técnicas de investigación, métodos, enfoques, conceptos o lenguaje cuantitativo o cualitativo en un solo estudio.

Merrian (2009, citado por Valenzuela y Flores, 2011) define como metodología cualitativa al enfoque que se deriva de filosofías constructivistas, fenomenológicas y de interaccionismo simbólico. Se trata de una investigación centrada en las personas que construyen sus mundos y en los significados que atribuyen a sus experiencias, a fin de entender el fenómeno de interés desde la perspectiva de los participantes y no desde la del investigador. En cuanto a la metodología cuantitativa, la define como aquella que en su faceta de ex post facto o llamada también no experimental, estudia información después del hecho y formula explicaciones del fenómeno de estudio.

Otra característica del estudio es ser transversal o de prevalencia, que, según García y Huerta (1998), este tipo de estudios mide simultáneamente la exposición a uno o varios factores, así como a la variable de interés, por lo que brinda información acerca de la frecuencia y distribución de diferentes factores. El indicador para cuantificar la frecuencia es la prevalencia. Agregan que, dado que la medición se realiza en un período único, breve y bien limitado hace que el costo de estos estudios no sea tan alto. La parte descriptiva del estudio según Aránguiz, García, Rojas, Salas, Martínez y Mac (2010) es porque se trabaja sobre realidades de hecho y su característica fundamental es la de presentar una interpretación correcta, la cual puede incluir los siguientes tipos de estudios: encuestas, casos, exploratorios, causales, de desarrollo, predictivos, de conjuntos y de correlación.

\subsection{Preguntas y objetivos}

De manera conjunta, se plantearon los siguientes objetivos a cubrir en la investigación:

a) Describir parte de las características y prácticas de un profesor que obtiene una evaluación del desempeño docente sobresaliente.

b) Describir parte de las características y prácticas de un profesor que obtiene una evaluación del desempeño docente deficiente.

c) Establecer una metodología que permita retroalimentar a los profesores de los resultados de la evaluación docente en función de las características positivas, para mejorar su desempeño.

La pregunta que se definió para esta investigación es la siguiente: ¿Cuál es la relación entre las características personales y de práctica docente con los resultados de la evaluación del desempeño de los profesores por parte de los estudiantes?

\subsection{Tipo de investigación e hipótesis}

La investigación se desarrolló con un enfoque cuantitativo (Stake, 2010) de tipo no experimental; es decir, sin control de la variable independiente y técnica de análisis de datos descriptiva. Se presentaron también las hipótesis siguientes: 
Ho = Hipótesis nula. No existe relación entre el constructo características personales y el constructo de práctica docente con el resultado de la evaluación del desempeño docente.

Ha $=$ Hipótesis alterna . Existe relación entre el constructo características personales y el constructo de práctica docente con el resultado de la evaluación del desempeño docente.

El constructo "características personales" comprende las variables siguientes:

- Edad

- Género

- Grado de Estudios

- Experiencia Profesional

- Vinculación (si el profesor realiza actividades profesionales fuera de la institución escolar)

El constructo "prácticas docentes" comprende las variables siguientes:

- Promedio de grupo

- Cantidad de alumnos reprobados

- Cantidad de faltas registradas

- Semestre del curso

- Innovación Educativa (uso de la tecnología, estrategias didácticas)

\subsection{Justificación}

El propósito de realizar esta investigación fue obtener información que pudiera contribuir a retroalimentar, desde una perspectiva diferente, a los profesores de un departamento académico en la universidad privada en la que se realizó este estudio y que, semestre a semestre, son evaluados en su desempeño docente por los alumnos. Los maestros siguen de cerca el desarrollo de este sistema evaluativo y se preguntan qué prácticas docentes son empleadas por los profesores que resultan evaluados de forma sobresaliente. Así mismo, para el director de un departamento académico, es importante conocer qué variables inciden en el desempeño de sus profesores, con el fin de apoyarlos para mejorarlo.

Además, en este caso de estudio se realizó una investigación de enfoque mixto para comprender una parte del fenómeno de la evaluación del desempeño docente. Asimismo, el caso buscó profundizar desde la perspectiva de los diferentes actores, como son los profesores, alumnos y directivos del Departamento Académico de Contabilidad y Finanzas de la universidad donde se realizó el estudio.

Se considera que este enfoque mixto es el idóneo para esta investigación porque permite obtener información y extraer conocimiento de las técnicas y herramientas cualitativas y cuantitativas, además de contrastar los resultados para aproximarnos a conclusiones más acertadas.

\subsection{Participantes}

Los participantes en esta investigación fueron profesores y directivos del Departamento Académico de Contabilidad y Finanzas de una universidad privada ubicada al norte de México y alumnos pertenecientes a la misma universidad. Se utilizó la información 
generada por los cuestionarios de evaluación de desempeño de profesores aplicadas; es decir, 9300 encuestas durante los semestres de los años 2015 y 2016. Estos datos representaron uno de los elementos sólidos del estudio, aportando a la viabilidad, ya que se encuentran debidamente organizados y estructurados en una base de datos construida expresamente para esta investigación. Se consideraron además algunas variables que definen las características personales y las de práctica docente. También se realizaron entrevistas a alumnos en semestres actuales, se llevaron a cabo observaciones en clases y se hicieron grupos de enfoque con alumnos para hablar sobre las encuestas que ellos responden sobre sus profesores cada final de semestre.

La población de profesores en el Departamento Académico de Contabilidad y Finanzas al momento del estudio era de 20 de tiempo completo y de 60 de tiempo parcial. Del total de 80 docentes y, mediante una muestra dirigida en función de sus atributos de desempeño docente, se seleccionaron 6 para entrevista y 6 para observaciones. Dentro de cada grupo de 6 profesores, se ubicaron 2 de desempeño sobresaliente, 2 de desempeño promedio y 2 de desempeño por debajo del promedio.

\subsection{Instrumentos}

Para esta investigación se contó con acceso a la base de datos de los resultados de las evaluaciones de desempeño realizadas por los estudiantes de los cursos de contabilidad y finanzas de los últimos tres semestres. Además, se obtuvo el acceso a información de carácter personal, currículums y promedios de calificaciones de los grupos donde los maestros del estudio desempeñaron su labor. Por otro lado, se utilizaron también los siguientes instrumentos:

Cuestionarios. Es un conjunto de preguntas estructuradas, enfocadas y estandarizadas que se responden en un formulario impreso o a través de medios electrónicos (Valenzuela y Flores, 2011). Como ya se mencionó, se utilizó la información de, aproximadamente, 9300 cuestionarios diseñados para la evaluación del desempeño docente de los alumnos a cada uno de sus profesores en los semestres enero-mayo y agosto-diciembre de los años 2015 y 2016. Dentro de la institución de estudio, estos cuestionarios son conocidos como ECOA, que es un instrumento institucional con confiabilidad, validez y consistencia. A pesar de que no se cuenta con el valor de confiabilidad preciso, es un instrumento que se ha usado consistentemente durante varios años (ver Apéndice C). Cada pregunta se califica del 1 al 5 donde el 1 significa el mejor desempeño y el 5 el peor desempeño. La información fue procesada de acuerdo a un ordenamiento descriptivo de los participantes de acuerdo a la edad, semestre y género. Además, se utiliza el promedio de calificaciones obtenido por los profesores, llamado Percentil, para establecer su desempeño y ubicarlo como profesor a ser recomendado por los alumnos, profesor que crea reto intelectual, profesor motivador o profesor inspirador.

Elizalde y Reyes (2008) hablan sobre la evaluación docente basada en la evaluación de los alumnos, mencionan que es uno de los modelos más utilizados y de más antigüedad. Además, "sirve para retroalimentar el trabajo dentro del aula. Si existe consistencia de los juicios de los alumnos a través del tiempo y entre grupos respecto a un mismo profesor, es posible decir que es un modelo confiable" (Elizalde y Reyes, 2008).

Entrevistas. Se realizaron entrevistas a profesores y a directivos. Estas fueron semiestructuradas con siete preguntas abiertas y dejando espacios de libre expresión para 
captar información valiosa y particular de cada profesor. Se utilizó un documento de confidencialidad para solicitar consentimiento para grabar. Se realizaron seis entrevistas en total, dos a profesores con resultados altos de evaluación, dos a profesores con resultados bajos y dos a profesores con resultados intermedios. Los profesores fueron de planta y cátedra. Se realizaron también dos entrevistas a Directivos del Departamento Académico acerca de los resultados obtenidos por los profesores y la utilidad del programa de evaluación.

Observaciones. Es una técnica de recolección de datos que puede ser utilizada en las investigaciones cuantitativa y cualitativa. El tipo de observación naturalista está asociada con la antropología y la psicología; es usada por los investigadores para adentrarse en los contextos a fin de comprender la cultura y procesos de los grupos (Valenzuela y Flores, 2011). Se llevó a cabo seis observaciones de clases completas impartidas por los maestros. Se observaron tres clases de maestros de los mejor evaluados y tres clases de maestros menos favorecidos en su evaluación. Las observaciones se centraron en: a) el estilo de comunicación verbal y no verbal del profesor, b) participaciones de los alumnos, c) ambiente de confianza y d) motivación.

Grupos de enfoque. Es un conjunto de entrevistas dirigidas por un moderador a un grupo pequeño de personas y con una temática en común. Típicamente son grupos de seis a ocho personas y con un nivel de conocimiento del tema similar (Morgan, 1997). En este estudio se realizaron dos grupos de enfoque con alumnos, en grupos de 12 a 16 y moderados por el investigador. La muestra se seleccionó de forma aleatoria. Esta actividad se llevó a cabo en un aula especialmente diseñada para esta técnica con la tecnología adecuada, como equipo de grabación, mobiliario, lay out de distribución y condiciones de privacidad y sonoridad.

\subsection{Estrategias de análisis de datos, validez y confiabilidad}

Al ser esta una investigación con enfoque mixto, el análisis de la información fue también mixto, procesada con enfoque cuantitativo y cualitativo. Para la parte cuantitativa se utilizó el paquete computacional Statgraphics. Mientras que, para la parte cualitativa se utilizó el software Atlas.ti.

La validez de un instrumento consiste en que mida lo que tiene que medir (autenticidad) (Corral, 2009) y responda a la pregunta: ¿con qué fidelidad corresponde el universo o población al atributo que se va a medir? Respecto a la confiabilidad, esta debe responder a la pregunta: ¿con qué exactitud los ítems, reactivos o tareas representan al universo de donde fueron seleccionados? Es decir, la confiabilidad designa la exactitud con que un conjunto de puntajes de pruebas mide. Existen instrumentos utilizados para recabar datos que, por su naturaleza, no ameritan el cálculo de la confiabilidad, como son: entrevistas, escalas de estimación, listas de cotejo, guías de observación, hojas de registros, inventarios, rúbricas, entre otros. Sin embargo, se requiere estimar o comprobar su validez (Cisterna Cabrera, 2005). En el caso particular de la encuesta ECOA y de la cual se ha tomado y procesado gran cantidad de información para este estudio, al ingresar el alumno al portal de la encuesta, se entiende que es un instrumento validado para este fin, sin embargo, se aclara que se busca que el alumno la conteste con objetividad y honestidad.

Además de asegurar la validez y confiabilidad de los instrumentos para que el estudio sea riguroso y consistente, se emplearon, donde fuera aplicable, las siguientes técnicas de los 
autores Valenzuela y Flores (2011): Triangulación de datos, de metodología y con la teoría y verificación con los participantes. De esta forma, se contrastaron los datos obtenidos entre los diferentes sujetos participantes en la investigación, confirmando los resultados.

\section{Resultados}

Para el manejo de los datos y el procesamiento de la información, es conveniente realizar categorizaciones. Estas permiten observar similitudes o discrepancias, relaciones, conjunciones u oposiciones. De tal manera que emerja información significativa para el investigador y el estudio. Valenzuela y Flores (2011) afirman que debe quedar muy claro que las categorías son abstracciones derivadas de los datos, no los datos por sí mismos.

Retomando la pregunta de investigación planteada y los objetivos, se da continuidad a encontrar las diferentes respuestas, utilizando dos categorías definidas de estudio para establecer una estructura de información. La categoría uno es: Prácticas y características que favorecen el desempeño sobresaliente; y la categoría dos: Prácticas y características que no favorecen el desempeño sobresaliente. Posteriormente, las prácticas y características obtenidas bajo los diferentes enfoques se analizan y contrastan para su interpretación final.

\subsection{Discusión de resultados con respecto a la pregunta de investigación. Enfoque cuantitativo}

La base informativa aquí analizada fue el producto de aproximadamente 9,400 encuestas realizadas por alumnos matriculados de todos los semestres en las carreras académicas que ofrece el Departamento Académico de Contabilidad y Finanzas de una universidad privada. El instrumento se aplicó en 469 grupos con 56 distintos profesores en los semestres enero-mayo y agosto-diciembre de los años 2015 y 2016.

La investigación aquí realizada se basó en un estudio factorial que, de acuerdo a Ferrando y Anguiano-Carrasco (2010), es un modelo estadístico que representa las relaciones entre un conjunto de variables y que, además, estas relaciones pueden explicarse a partir de una serie de variables latentes denominadas factores, siendo el número de factores substancialmente menor que el de variables. Donde también, este tipo de modelos se obtienen de las ideas básicas de los modelos de regresión lineal y de correlación parcial. Este autor basó su publicación en investigaciones psicológicas, donde el análisis factorial analiza un conjunto de variables observables (ítems, subtests o tests) y cada una de las cuales puede considerarse como un criterio.

Los datos se adecuaron y normalizaron en una base de Excel y, posteriormente, se trataron y analizaron usando el programa estadístico Statgraphics. Se buscó encontrar relación entre la variable definida como dependiente: Evaluación del Desempeño Docente (EDD), que es la calificación de alumnos a profesores al final del semestre y que evalúa temas como metodología y actividades de aprendizaje, comprensión de conceptos en términos de su aplicación en la práctica, interacción con el profesor y la asesoría recibida, sistema de evaluación, reto intelectual, guía del aprendizaje y profesor como inspirador. Y la relación con las variables definidas como independientes que son: experiencia profesional del docente, grado último de estudios del profesor, número de alumnos que reprueba al final del curso, su género y edad. 
El paquete Statgrapichs realiza, mediante algoritmos de suma de cuadrados y modelos matriciales, el planteamiento de una ecuación con los factores que mayor contribución aportan la variable dependiente, eliminado de todos los demás factores. La ecuación resultante con sus coeficientes es la siguiente:

Modelo de ecuación general es:

$$
\Upsilon=K+c i+c 2+c 3 \ldots+C n
$$

Ecuación para nuestro estudio particular:

$$
E D D=K+(1,51) A+(2,04) B+(21,01) C+(11,8) D+(1,42) E
$$

Donde el factor A es la experiencia del profesor, la tenga o no en la parte laboral. El factor $\mathrm{B}$ es grado del profesor, maestría o doctorado. El factor $\mathrm{C}$ es el número de alumnos reprobados por grupo, hasta dos o más de dos. El factor $\mathrm{D}$ es el género, masculino o femenino. Y finalmente el factor E, indica la edad de los profesores, definida por los siguientes rangos: de 35 a 45 años, de 46 a 55 años y de 56 a 70 años.

Los estadísticos descriptivos particulares que dan base al presente estudio se presentan en el cuadro 1.

Cuadro 1. Resultados estadísticos descriptivos del estudio

\begin{tabular}{lc}
\hline Número de grupos escolares analizados & 465 \\
Promedio de calificaciones & 8,87766 \\
Desviación estándar & 0,877417 \\
Coeficiente de variación & $9,88343 \%$ \\
Error estándar & 0,0406892 \\
Calificación mínima (valor mínimo) & 2,83 \\
Calificación máxima (valor máximo) & 10,0 \\
Rango (resta del valor mayor al menor) & 7,17 \\
\hline Fuente: Datos procesados del paquete Statgraphics Plus.
\end{tabular}

Fuente: Datos procesados del paquete Statgraphics Plus.

Este cuadro 1 proporciona los datos que dan soporte y estructura del análisis de los datos presentados, como el número de grupos a los que se les hizo la encuesta y que aparecen en este análisis estadístico. Siendo 465 grupos con un promedio de 20 alumnos, suma alrededor de 9300 alumnos encuestados. También se observa que el promedio de calificaciones emitidas es de 8,87. Así como el coeficiente de variación de los datos que se expresa en porcentaje, 9,8\% para este caso, donde no hace ver la variación entre la media y nuestra variable que es la calificación otorgada al docente. Indicando menor del 10\%, lo que permite congruencia estadística para desarrollar el estudio.

El cuadro 2 muestra cinco de las variables que afectan al constructo Evaluación del desempeño docente (EDD), analizadas en el programa Statgraphics. Resultando estadísticamente significativas con el 95\% de confianza, siendo el factor $\mathrm{C}$, número de alumnos reprobados por curso y el factor $\mathrm{D}$, el género. Esto se debe a que los factores antes mencionados son inferiores a 0,005, por lo tanto, resultan en un efecto estadísticamente significativo. 
Cuadro 2. Análisis de varianza de efectos principales. - Tipo III Suma de cuadrados, con intervalos de confianza del 95,0\%

\begin{tabular}{lccccc}
\hline FACTOR & $\begin{array}{c}\text { SUMA DE } \\
\text { CUADRADOS }\end{array}$ & $\begin{array}{c}\text { GRADOS } \\
\text { DE } \\
\text { LIBERTAD }\end{array}$ & $\begin{array}{c}\text { CUADRADO } \\
\text { MEDIO }\end{array}$ & $\begin{array}{c}\text { COEFICIENTE } \\
\text { F }\end{array}$ & $\begin{array}{c}\text { VALOR P } \\
\text { SIGNIFICACIÓN } \\
\text { ESTADÍsTICA }\end{array}$ \\
\hline $\begin{array}{c}\text { A (experiencia } \\
\text { del profesor) }\end{array}$ & 1,07148 & 1 & 1,07148 & 1,51 & 0,2203 \\
$\begin{array}{c}\text { B (grado del } \\
\text { profesor) }\end{array}$ & 1,45324 & 1 & 1,45324 & 2,04 & 0,1536 \\
$\begin{array}{c}\text { C (número de } \\
\text { alumnos } \\
\text { reprobados) }\end{array}$ & 14,9413 & 1 & 14,9413 & 21,01 & 0,0000 \\
$\begin{array}{l}\text { D (género) } \\
\text { E (edad) }\end{array}$ & 8,39131 & 1 & 8,39131 & 11,80 & 0,0006 \\
\hline
\end{tabular}

Fuente: Datos arrojados del paquete Statgraphics Plus.

Posteriormente, se corrió el análisis del modelo para conocer si existen interacciones significativas entre los factores, encontrándose las siguientes: AE, experiencia profesional con la edad; BD, el grado con el género; BE el grado con la edad y, finalmente, DE que es el género con la edad (ver cuadro 3).

Cuadro 3. Análisis de varianza para efectos con interacciones

\begin{tabular}{cccccc}
\hline FACTOR & $\begin{array}{c}\text { SUMA DE } \\
\text { CUADRADOS }\end{array}$ & $\begin{array}{c}\text { GRADOS } \\
\text { DE } \\
\text { LIBERTAD }\end{array}$ & $\begin{array}{c}\text { CUADRADO } \\
\text { MEDIO }\end{array}$ & $\begin{array}{c}\text { COEFICIENTE } \\
\text { F }\end{array}$ & $\begin{array}{c}\text { VALOR P } \\
\text { (SIGNIFICACIÓN } \\
\text { ESTADÍSTICA }\end{array}$ \\
\hline AB & 1,0294 & 1 & 1,0294 & 1,63 & 0,2026 \\
AC & 0,706665 & 1 & 0,706665 & 1,12 & 0,2909 \\
AD & 0,00198931 & 1 & 0,001989 & 0,00 & 0,9553 \\
AE & 11,8792 & 2 & 5,93962 & 9,40 & 0,0001 \\
BC & 0,0000020 & 1 & 0,000002 & 0,00 & 0,9986 \\
BD & 9,45042 & 1 & 9,45042 & 14,95 & 0,0001 \\
BE & 5,70103 & 2 & 2,85052 & 4,51 & 0,0115 \\
CD & 1,48638 & 1 & 1,48638 & 2,35 & 0,1259 \\
CE & 1,82785 & 2 & 0,913925 & 1,45 & 0,2367 \\
DE & 13,0832 & 2 & 6,5416 & 10,35 & 0,0000 \\
\hline
\end{tabular}

Fuente: Datos procesados y emitidos por el paquete Statgraphics Plus.

Se observa que el factor que más influye significativamente como interacción es la edad del profesor. Aunque no arroja resultados específicos del nivel de la edad, refleja que es estadísticamente influyente como interacción. Clave que puede ser un tópico a desarrollar para futuras investigaciones.

\subsection{Discusión de las características y prácticas de los profesores y su impacto en la evaluación del desempeño docente. Con enfoque cualitativo}

La parte de la investigación cualitativa de este estudio se desarrolló en la misma universidad privada también con alumnos y profesores de las carreras profesionales que ofrece el Departamento Académico de Contabilidad y Finanzas. Se entrevistó a tres profesores de tiempo completo con experiencia de más de 20 años en cátedra, dos de ellos con nivel de doctorado y uno con dos maestrías, además de múltiples cursos y diplomados que robustecen su currículum y los tres con experiencia internacional docente. Se realizaron observaciones con profesores en tres grupos de, aproximadamente, 30 alumnos cada uno. Estos profesores fueron seleccionados con base en que el profesor uno resultó 
altamente favorecido en las encuestas el profesor dos medianamente favorecido y el profesor tres, que resultó menos favorecido; sin hacerlo saber al investigador hasta concluir la recolección de datos para evitar el sesgo. Cabe mencionar que la trayectoria académica de estos profesores es sobresaliente. Los dos primeros con más de 20 años en la docencia y grado de maestría y el tercero con menos años en la docencia, pero con reconocida experiencia en el aspecto laboral.

Con respecto a la observación de campo, esta se realizó en tres grupos de aproximadamente 15 alumnos cada uno. Los dos paneles de entrevistas, focus group con total de 9 alumnos participantes, se realizaron a estudiantes de carreras administrativas de los últimos cuatro semestres.

Como actividad inicial, se realizó la transcripción de la información obtenida con los diferentes instrumentos de investigación que se aplicaron: entrevistas a profesores, observación de clases y focus group con alumnos.

Para facilitar el entendimiento de los datos en este enfoque metodológico, estos se organizaron en unidades o categorías para mejor visualización y comprensión, permitiendo así encontrar patrones o discrepancias entre ellos y seleccionar la información significativa que a los fines y objetivos de la investigación compete. La primera categoría es: Las características y prácticas que impactan favorablemente en la Evaluación del Desempeño Docente (EDD). La segunda categoría es: Las características y prácticas que impactan desfavorablemente en la EDD.

Las características se seleccionaron de acuerdo al número de comentarios y evidencias redundantes encontradas con los instrumentos. La influencia de la característica que favorece o desfavorece se muestra clasificada como alta, media o baja en el cuadro 4 . El orden de presentación no es escalonado y los resultados encontrados fueron que se muestran a continuación.

Con el estudio cualitativo y producto de los múltiples recursos de este enfoque, se logró conformar y concluir que el modelo de un profesor inspirador para los alumnos de las carreras económicas y financieras, debe presentar las características siguientes:

Docente atento y cortés con sus alumnos, no presentando momentos de indiferencia. Con experiencia práctica fuera del ámbito escolar en la materia que esté impartiendo. Que muestre ejemplos y casos prácticos que relacionen la teoría con la práctica. Con pensamiento flexible para adaptarse a las muy diferentes generaciones que ingresan cada semestre. Que deje ver su lado humano al interesarse en aspectos no únicamente escolares del alumno. Bien vestido y creativo al diseñar ambientes de aprendizaje. $\Upsilon$ por último, y situación que requeriría más trabajo colaborativo entre alumnos y maestros, con interés por disminuir el indice de reprobación. 
Cuadro 4. Categorización de hallazgos con la metodología cualitativa

\begin{tabular}{|c|c|c|}
\hline $\begin{array}{c}\text { CATEgORÍA No.1: CARACTERíSTICAS Y } \\
\text { PRÁCTICAS QUE IMPACTAN } \\
\text { FAVORABLEMENTE EN LA EDD }\end{array}$ & $\begin{array}{c}\text { CATEGORÍA NO.2: } \\
\text { CARACTERÍSTICAS Y PRÁCTICAS } \\
\text { QUE NO IMPACTAN } \\
\text { FAVORABLEMENTE EN LA EDD }\end{array}$ & $\begin{array}{l}\text { GRADO DE } \\
\text { INFLUENCIA }\end{array}$ \\
\hline $\begin{array}{l}\text { Características personales del profesor: } \\
\text { atento, cortés, con variantes en el tono de } \\
\text { voz, adecuada postura corporal }\end{array}$ & Indiferencia hacia los alumnos & Alta \\
\hline $\begin{array}{l}\text { Experiencia práctica en la materia que } \\
\text { está impartiendo el profesor y dominio de } \\
\text { su contenido }\end{array}$ & $\begin{array}{l}\text { Que el profesor imparta una } \\
\text { materia sin experiencia práctica }\end{array}$ & Alta \\
\hline Profesor con bajo índice de reprobados & $\begin{array}{l}\text { Profesor con alto índice de } \\
\text { reprobados }\end{array}$ & Alta \\
\hline $\begin{array}{l}\text { Interés personal por alumnos de temas } \\
\text { extraescolares, generando relación de } \\
\text { confianza personal. }\end{array}$ & Poco interés & Alta \\
\hline $\begin{array}{l}\text { Relación del contenido teórico de la } \\
\text { materia con casos prácticos aplicables y } \\
\text { su futura aplicación en su vida } \\
\text { profesional }\end{array}$ & & Alta \\
\hline $\begin{array}{l}\text { Flexibilidad hacia las características que } \\
\text { presentan cada diferente generación }\end{array}$ & No flexibilidad & Alta \\
\hline $\begin{array}{l}\text { Tipo de vestimenta y arreglo personal lo } \\
\text { más cuidada posible }\end{array}$ & $\begin{array}{l}\text { Aspecto descuidado, mal vestido y } \\
\text { desarreglado }\end{array}$ & Media \\
\hline $\begin{array}{l}\text { El currículum académico del profesor, } \\
\text { enfocado al grado de doctorado o } \\
\text { maestría. Además de otras capacitaciones } \\
\text { y actualizaciones referentes a la materia } \\
\text { impartida }\end{array}$ & & Media \\
\hline $\begin{array}{l}\text { Diseño y construcción de ambientes de } \\
\text { aprendizaje }\end{array}$ & $\begin{array}{l}\text { La poca variedad de las técnicas de } \\
\text { enseñanza }\end{array}$ & Media \\
\hline $\begin{array}{l}\text { Capacidad de recibir crítica y desarrollar } \\
\text { autocrítica }\end{array}$ & No reconocer errores & Media \\
\hline Dirigirse a los alumnos por su nombre & $\begin{array}{l}\text { No recordar el nombre de sus } \\
\text { alumnos }\end{array}$ & Media \\
\hline $\begin{array}{l}\text { Vincular a los alumnos con el entorno en } \\
\text { proyectos finales o parciales }\end{array}$ & & Media \\
\hline Presentar retos a los alumnos & & Media \\
\hline $\begin{array}{l}\text { Fomentar el trabajo colaborativo con los } \\
\text { alumnos y cambiar roles en los equipos }\end{array}$ & & Baja \\
\hline Establecer disciplina en clase & & Baja \\
\hline Sentido del humor & & Baja \\
\hline
\end{tabular}

Fuente: Observación de campo.

\section{Discusión y conclusiones}

Retomando el objetivo principal de la presente investigación, que es describir las características y prácticas de un profesor que le llevan a obtener una evaluación de su desempeño docente como sobresaliente, se puede concluir que, a partir de los datos obtenidos por métodos cualitativos y cuantitativos, se ha logrado describir dichas características y prácticas, pretendiendo que esta sea un aporte significativo para la construcción del conocimiento en este campo del saber del área de la educación. 


\subsection{Con respecto a las características y prácticas que influyen favorablemente en la evaluación del desempeño docente}

En respuesta a la pregunta de investigación planteada, ¿cuál es la relación entre las características personales y de práctica docente con los resultados de la evaluación del desempeño de los profesores por parte de los estudiantes pertenecientes a la universidad de estudio?, se puede concluir que, efectivamente, existe una relación directamente proporcional. Además, se logró identificar las características y prácticas de mayor significancia para esta relación, mismas que se mencionan en el siguiente párrafo.

Con la riqueza de la información producida al evaluar a 469 grupos, con 56 distintos docentes que arrojaron, aproximadamente, 9300 encuestas procesadas durante los años 2015 y 2016, y analizando ciertas características de los profesores como fueron su género, edad, número de alumnos que reprueban por semestre y si contaban con experiencia fuera del ámbito escolar o relacionada con la materia impartida, estadísticamente se concluye que influye significativamente si un profesor tiene un bajo índice de reprobación en su grupo y si su género es femenino, en ser favorablemente evaluado.

Con respecto a la influencia de la cantidad de alumnos reprobados, es necesario indagar más, si esto significa que un menor número de reprobados implica un mayor interés del profesor en el aprendizaje de sus alumnos y no necesariamente que el profesor sea "fácil" o de "poca exigencia". Aquí es necesario relacionar los resultados con otras variables del desempeño del profesor. Posteriormente, se buscó encontrar características que, combinadas, influyeran significativamente en una evaluación favorable, resultando que la edad, se presenta como factor más influyente. A mayor edad mejor evaluado en forma combinada.

De los resultados presentados en el cuadro 4, se pudo observar que los alumnos aprecian que sus maestros cuenten con características y cualidades que los ayuden a relacionarse con sus estudiantes, que creen un ambiente de confianza y respeto. Además, reconocen también a aquellos profesores que no sólo conocen de su materia, sino que tienen experiencia llevándola a la práctica. Por el contrario, un profesor que tiene un alto número de reprobados o que no se interesa por sus alumnos, tiende a recibir una mala calificación. Algunos otros aspectos que se consideraron en las pruebas, pero que para los alumnos no resultan tan necesarias en sus profesores, son, por ejemplo, su sentido del humor, que recuerde el nombre de los alumnos o que planee actividades en equipo. En resumen, según los resultados presentados en este cuadro, los alumnos desean, a grandes rasgos, que sus profesores conozcan y sepan implementar su materia y que, además, sepan relacionarse con sus estudiantes.

\subsection{Con respecto a la evaluación del desempeño como un instrumento de mejora continua para la práctica docente}

Haciendo referencia al objetivo planteado de la metodología de retroalimentación al profesor de las características personales y prácticas docentes que le pueden llevar a obtener una calificación más favorable con sus alumnos, se propone un sistema de gestión basado en el ciclo de Deming (Deming, 1989). Las fases del ciclo son: Planear, Hacer, Verificar y Actuar. En la fase de Planear se realiza la documentación; es decir, se estructura el plan de introducir las nuevas prácticas de enseñanza y modificación de características personales. En la fase de Hacer, se ejecuta el plan y se generan evidencias documentadas 
de lo realizado. En la fase de Verificar se miden los resultados obtenidos. Finalmente, en la fase final de Actuar, se replantea lo primeramente documentado con base en los resultados obtenidos. Como se observó, es un ciclo donde, en cada cierre, se produce madurez en el proceso de enseñanza.

La evaluación de desempeño docente, debe ser un proceso que pueda abordarse desde diferentes perspectivas. La opinión de los alumnos, siempre será valiosa pues ellos son los principales beneficiarios del quehacer docente. Sin embargo, la opinión de los colegas y de los superiores, en combinación con un ejercicio de autoevaluación alineado a un plan de desarrollo personal, constituyen insumos que hacen que el profesor sienta como propia la evaluación, no como un mecanismo externo de control, sino como un medio para su desarrollo y crecimiento personal y profesional.

\subsection{Con respecto a futuras investigaciones sobre evaluación del desempeño docente}

Será interesante indagar a mayor profundidad sobre las variables de género y cantidad de alumnos reprobados, las cuales resultaron estadísticamente significativas con la evaluación del desempeño docente desde la perspectiva de los alumnos. Puede pensarse en las características que derivan del género femenino como cualidades de carácter que un profesor, independientemente de su género, podría desarrollar. Y, además, indagar por qué esas características son reconocidas como favorables para ser un buen profesor desde la perspectiva de los alumnos.

Con respecto al índice de reprobación, sería interesante comprender más allá del número de alumnos reprobados y aprobados como mero indicador de desempeño, o de exigencia, o de falta de ella, si hay un interés genuino por parte del profesor de lograr el aprovechamiento de todos sus alumnos, mediante una cuidadosa planificación de contenidos, evaluaciones y retroalimentación, antes del desenlace del fin de cursos.

Finalmente, es necesario tomar conciencia de que las generaciones actuales son altamente reflexivas con respecto al desempeño de sus profesores. Cuestionan el porqué de cada actividad y la utilidad de esta en su vida profesional futura. Sin mencionar que tienen acceso a múltiples fuentes de información para obtener datos sobre el currículum académico de su profesor, su experiencia laboral y la pertinencia y validez de los contenidos que enseña en su materia, por lo que la exigencia hacia el profesor actual es cada vez mayor y esto resulta en un reto para las instituciones educativas. Hoy más que nunca, los estudiantes requieren de un profesor más allá de la exposición de contenidos, lo que la tecnología, el Internet y las redes sociales no pueden proveerles: experiencia, guía e inspiración para lograr el éxito en su preparación académica y en su vida profesional.

\section{Referencias}

Achilli, E. (1988). La práctica docente: una interpretación desde los saberes del maestro. Cuadernos de Antropología Social, 1(2), 5-18.

Alvarado, E., Canales, F. y Pineda, E. (1994). Metodología de la Investigación.Washington, DC: Organización Panamericana de la Salud.

Angulo J. F. y Blanco, N. (1994). Teoría y desarrollo del curriculum. Málaga: Aljibe. 
Aránguiz, H., García, V., Rojas, S., Salas, C., Martínez, R., y Mac Millan, K. (2010). Estudio descriptivo, comparativo y correlacional del estado nutricional y condición cardiorrespiratoria en estudiantes universitarios de Chile. Revista Chilena de Nutrición, 37(1), 70-78. https://doi.org/10.4067/S0717-75182010000100007

Arbesú, M., y Rueda, M. (2003). La evaluación de la docencia desde la perspectiva del propio docente. REencuentro. Análisis de Problemas Universitarios, 36, 56-64.

Cantú D. J. H. (2011). Desarrollo de una cultura de calidad. Ciudad de México: McGraw-Hill Interamericana.

Castillo Arredondo, S. (2010). La práctica de la evaluación educativa: materiales e instrumentos. Madrid: Pearson.

Castillo Arredondo S. y Cabrerizo Diago, J. (2010). Evaluación educativa de aprendizajes y competencias. Madrid: Pearson Educación.

Cisterna Cabrera, F. (2005). Categorización y triangulación como procesos de validación del conocimiento en investigación cualitativa. Theoria, 14(1), 61-71. https://doi.org/10.25267/Rueda.2015.07

Corral, Y. (2009). Validez y confiabilidad de los instrumentos de investigación para la recolección de datos. Revista Ciencias de la Educación, 33(19), 224-247. https://doi.org/10.15517/rcs.voi141.12480

Deming E. (1989). Calidad, productividad y competitividad. La salida de la crisis. Madrid: Editorial Díaz de Santos.

Díaz-Barriga, F. y Rueda M. B. (2000). La evaluación de la docencia perspectivas actuales. Ciudad de México: Paidós Educador.

Duncan, A. (2011). Duncan tells teachers: change is hard. Recuperado de https://www.ed.gov/news/speeches/change-hard

Elizalde Lora, L. y Reyes Chávez, R. (2008). Key Elements for the Evaluation of the Teaching Performance. RELIEVE, $1 O$ (spe), 1-13. https://doi.org/10.7203/relieve.10.1.4331

Ferrano, P. J. y Anguiano-Carrasco, C. (2010). El análisis factorial como técnica de investigación en psicología. Papeles del Psicólogo, enero-abril, 18-33. https://doi.org/10.15381/rinvp.v17i2.11269

Fierro, C., Fortoul, B. y Rosas, L. (1999). Transformando la práctica docente, una propuesta basada en la investigación-acción. Ciudad de México: Paidós.

García, C. M., y Vaillant, D. (2010). Desarrollo profesional docente: ¿Cómo se aprende a enseñar? Madrid: Narcea Ediciones.

García de la Torre, G. S., y Huerta Alvarado, S. G. (1998). Consideraciones metodológicas y análisis simple de los estudios transversales. Boletín Médico del Hospital Infantil de México, 55(6), 34856 .

Guadagni, A. A. (2015). El ranking de las universidades en el mundo y en américa latina. Centro de Estudios de la Educación Argentina, 39.

Johnson, B. y Onwuegbuzie, A. (2004). Mixed methods research: A research paradigm whose time has come. Educational Researcher, $\quad 33(7), \quad$ 14-26. https://doi.org/10.3102/0013189X033007014

Lukas J. y Santiago, K. (2014). Evaluación educativa (2a. ed.). Madrid: Alianza Editorial. 
Luna, E. y Torquemada, A. (2008). Los cuestionarios de evaluación de la docencia por los alumnos: balance y perspectivas de su agenda. REDIE. Revista Electrónica de Investigación Educativa, especial, 1-15. https://redie.uabc.mx/redie/article/view/201

Martínez, J. F. (2013). Combinación de mediciones de la práctica y el desempeño docente: consideraciones técnicas y conceptuales para la evaluación docente. Pensamiento Educativo: Revista de Investigación Educacional Latinoamericana, $50(1), \quad 4-20$. https://doi.org/10.7764/PEL.50.1.2013.2

Martínez González, A., Sánchez Mendiola, M. y Martínez Stack, J. (2010). Los cuestionarios de opinión del estudiante sobre el desempeño docente: Una estrategia institucional para la evaluación de la enseñanza en Medicina. REDIE. Revista Electrónica de Investigación Educativa, 12(1), 1-18. https://redie.uabc.mx/redie/article/view/247

Morgan, D. L. (1997). The focus group guidebook. Nueva York, NY: Sage.

Muñoz Cantero, J. M., Rios de Deus, M. P. y Abalde, E. (2002). Evaluación docente vs evaluación de la calidad. RELIEVE, Revista Electrónica de Investigación y Evaluación Educativa, 8(2), 103134. https://doi.org/10.7203/relieve.8.2.4362

Páramo, P. (2008). Factores psicosociales asociados a la evaluación del docente. Educación y Educadores, 11(1), 11-30. https://doi.org/10.5294/edu.2017.20.3.2

Pereira Pérez, Z. (2011). Los diseños de método mixto en la investigación en educación: Una experiencia concreta. Revista Electrónica Educare, 15(1), 15-29. https://doi.org/10.15359/ree.21-2.7

Rueda, M. (2008). La evaluación del desempeño docente en la universidad. REDIE. Revista Electrónica de Investigación Educativa, Especial. Recuperado de https://redie.uabc.mx/redie/article/view/196

Rueda, M. y Díaz-Barriga, F. (2011). La evaluación de la docencia en la universidad: perspectivas desde la investigación y la intervención profesional. Ciudad de México: Plaza y Valdés.

Rueda, M., Schmelkes, S. y Díaz-Barriga, Á. (2014). La evaluación educativa. Presentación del número especial de Perfiles Educativos 2013. La evaluación en la educación superior. Perfiles Educativos, XXXVI(145), 190-204. https://doi.org/10.1016/So185-2698(14)70645-2

Stake, R. E. (2010). Investigación con estudio de casos. Madrid: Ediciones Morata.

Tejedor, F. y García-Valcárcel, A. (2010). Evaluación del desempeño docente. Revista Española de Pedagogía, 247, 439-459. https://doi.org/10.13042/Bordon.2016.68205

Theall, M. y Franklin, J. (2000). Creating responsive student ratings systems to improve evaluation practice. New Directions for Teaching and Learning, 83, 45-105. https://doi.org/10.1002/tl.8308

Valenzuela González, J. R., y Flores Fahara, M. (2011). Fundamentos de investigación educativa: El proceso de investigación educativa. Revista Latinoamericana de Investigación en Matemática Educativa, 19(1), 99-124. https://doi.org/10.12802/relime.13.1914

Vásquez-Rizo, F. E., y Gabalán-Coello, J. (2012). La evaluación docente en posgrado: variables y factores influyentes. Educación y Educadores, 15(3), 445-460. 


\section{Apéndice A}

\section{Entrevistas realizadas a profesores (Transcripción)}

Entrevista No. 1, a profesor con grado de Doctor y ex directivo del departamento. Con 35 años de experiencia docente

1.- ¿Cómo calificaría su interés por enseñar?

Inicia en mi juventud, enseñando a mis cuatro hermanas, amigos y amigas y eso despertó mi interés. Tuve una maestra, María del Carmen de la Garza, primera mujer graduada en el Tec de Contador Público y una de las primeras también en obtener MBA (Master Business_Administration) en Indiana, EU. Yo también estudié una maestría en España y MBA en NY. Continuando con el la maestra Carmen, me invita a dar clases en el Tec. Desde que fui su alumno, la consideraba muy buena maestra, fue motivadora y con mucha paciencia. Por lo que decidí integrarme al Tec como profesor. Al principio traté de imitar su estilo de enseñanza, a lo largo de los años he desarrollado mi propio estilo de enseñanza y así llevo cerca de 35 años.

\section{2.- ¿Qué habilidades pedagógicas o prácticas considera necesarias para enseñar?}

Considero el sentir empatía con los alumnos, el dominio del contenido de la materia, hacerles ver la importancias de la clase que se imparte. Y no solo para el cumplimiento del programa de su carrera, sino la utilidad también en el ambiente laboral. Resumidas en competencias, actitudes de aprender, valores y resaltando el trabajo en equipo. También desde luego, la interacción con el alumno de temas no necesariamente de clases. Te comento que a mis clases siempre me gusta llegar temprano y charlar de otros temas con mis alumnos para conocer sus costumbres, pensamientos, entre otros temas. Considero también muy importante el buscar o propiciar que los mismos alumnos, descubran y se conecten con el motivo por el cual están estudiando lo que están estudiando.

3.- De esas habilidades, ¿̨cuáles considera influyen directamente en la calificación que el alumno otorga al profesor?

Todas las anteriores.

4.- ¿Qué otros factores considera que pueden incidir en el resultado de la evaluación?

La rigidez de horarios y actividades y en ocasiones de exámenes. El esmero por explicar y que el alumno entienda. Ser justo en las evaluaciones, nada fácil. Además que el maestro prepare la clase porque de contrario los alumnos siempre se dan cuenta.

\section{5.- ¿Qué recomendaría a un colega para mejorar su práctica docente?}

Culturalmente, en México ir a la escuela es una actividad triste. En EU observo que los alumnos van a divertirse. Lo comprobé con mis hijos. Por lo cual podrían los colegas, crear ambientes de enseñanza en las aulas más lúdicos. Mostrar a los alumnos información actualizada de la materia y ponerla en un contexto aplicable.

6.- Considera que el tipo de materia influye en la evaluación?

No.

7.- ¿Desea agregar otras aportaciones? 
$\mathrm{Si}$, considero que de los millenials evalúan o ponderan el peso de cada actividad a realizar y deciden hacerla o no. Además que ésta generaciones recientes le demandan más esfuerzo y competencias al momento de impartir la clase. Por lo que se requiere como profesor, encontrar "Diferentes formas de enseñar lo mismo". Por mi experiencia en otros países de Europa y EU, considera que ellos le dan más importancia a la preparación y socialización hacia la vida laboral que la cantidad de conocimientos que el alumno adquiera. Contrario en nuestro país.

\section{Entrevista No. 2, a profesor con grado de Doctor y ex directivo del departamento. Con más de 25 años de experiencia docente.}

1.- ¿Cómo calificaría su interés por enseñar?

Tengo el convencimiento que el trabajo de enseñar influye positivamente en las personas por lo que esta profesión me motiva a ser cada vez mejor. Me resulta absorbente y apasionante.

Cuando imparto clase, me se olvida del resto de las cosas. Vivo el enseñar como una película donde esta actividad demanda alta concentración y trabajo mental y eso resulta placentero. Como comentario adicional, he notado que cuando imparto clases duermo mejor que cuando no. Mi interés también es dejar un buen recuerdo en sus alumnos y no pasar inadvertido. Entre mejor desempeño como docente realice, se multiplica a la parte social.

\section{2.- ¿Qué habilidades pedagógicas o prácticas considera necesarias para enseñar?}

Lo más básico es la conexión que pueda hacer con los estudiantes. Porque es difícil enseñar cuando un alumno tiene miedo o no respeta al profesor. El conjunto de las habilidades pedagógicas nos debe llevar a lograr recuerdos y aprendizajes significativos en los alumnos para que cuando lo requieran, busque en su memoria, en sus apuntes, sepa a quién recurrir y lo puedan aplicar.

3.- De esas habilidades, ¿̇cuáles considera influyen directamente en la calificación que el alumno otorga al profesor?

Definitivamente todas y las más posibles, pero reitero muy importante ganarse la confianza de los alumnos. Identificar y conocer a cada uno de los alumnos, saludarlo en los pasillo y de preferencia por su nombre. Eso lo conecta con la clase.

4.- ¿Qué otros factores considera que pueden incidir en el resultado de la evaluación?

Muy importante hacer "liga" con los alumnos. Porque de lo contrario, si los alumnos ni el nombre del profesor se aprenden, menos recordarán los conceptos de la clase que les pueden servir en su vida profesional, aunque hayan aprobado la materia. Lograr la atención del alumno no es fácil porque está compitiendo con 4 ó 5 colegas por la atención de los alumnos.

Considero que difícilmente un profesor que es "blando", salga mal evaluado. Puede ser que no salga bien evaluado pero no mal. Es un relación de reciprocidad, si el maestro es exigente, el alumno así será en la evaluación. Si el maestro exige poco, es probable que el alumno lo califique bien sin mucha exigencia. 


\section{5.- ¿Qué recomendaría a un colega para mejorar su práctica docente?}

Principalmente que cubrieran los programas académicos. Que se ocuparan hacia un verdadero interés de que el alumno logre el aprendizaje, no aprobar por complacer. Trabajar con enfoque de largo plazo con los alumnos, no al corto plazo. Y trata de mantener al margen el riesgo de la vanidad intelectual por ser el profesor en el aula la máxima autoridad. Y reconocer que hay alumnos que son más listos que el profesor.

\section{6.- Otras aportaciones.}

En el cuestionario de evaluación del desempeño docente, ECOA, no le preguntan al alumno si de verdad aprendió y si merece pasar la materia. Aquí observo un área de oportunidad importante. Porque considero que el objetivo primordial es el aprendizaje y claro, también muy importante es el alumno se siente cómodo pero no por encima del aprendizaje. Me gusta esta analogía, de realizar un viaje. Llegar al destino es lograr aprendizaje y el grado de comodidad como se realizó el viaje, sería lo que actualmente se evalúa más en el ECOA. Otra área de oportunidad en la metodología de las evaluaciones podría ser, por ejemplo, evaluar al principio del curso y al final pero por personas independientes.

Respecto al ofrecimiento de oferta educativa, observo que la en la actualidad hay una lucha por conservar y atraer alumnos a las escuelas privadas. Con la posible práctica de caer en la complacencia hacia el alumno. Y esto desde los niveles básicos de educación hasta la educación profesional. Por lo que la exigencia del maestro al alumno se puede ver comprometida. Énfasis en que el alumno aprenda, no que obtenga 10 ó 9 u 8 de calificación. Que pueda salir con promedio de 7 pero que sea competitivo laboralmente. También considero que se deberían implantar mecanismos para detectar si un alumno tiene fallas de aprendizaje en el transcurso de su carrera, y mecanismos para corregirlas

\section{7.- Considera que el tipo de materia influye en la evaluación?}

No.

\section{Entrevista No. 3, a profesora con maestrías y post grados. Con más de 25 años de experiencia docente.}

\section{1.- ¿Cómo calificaría su interés por enseñar?}

Me gusta mucho enseñar. Desde joven me inicio dando catecismo. Siento una realización cuando imparto clase y puedo observar la cara de los alumnos que le están entendiendo. Además estoy en aprendizaje constante porque varios de los alumnos trabajan en despachos o en sus empresas familiares.

\section{2.- ¿Qué habilidades pedagógicas o prácticas considera necesarias para enseñar?}

Principalmente vocación y formación académica. Conocimiento amplio de la materia, así como contar con experiencia en la práctica laboral. Liderazgo, ser un profesor motivador e inspirador. Llevar un programa y ser estructurado. Ser flexible, adaptarse a los cambios de cada nueva generación. Contar con técnicas didácticas. Y desde luego, ser feliz dando clases.

3.- De esas habilidades, ¿̇cuáles considera influyen directamente en la calificación que el alumno otorga al profesor? 
El conocimiento amplio de la materia a impartir. Desplegar este conocimiento de la mejora manera posible. Bien preparada y estructurada la clase y presentar casos prácticos. La experiencia personal en empresas. Influye el impartir diplomados o clases en otras universidades. Viajes con los alumnos, nacionales y extranjeros. Utilizar tecnología en el salón de clases. Actividades interactivas o colaborativas. No únicamente resolver los casos de los libros, sino conectarlos a una aplicación práctica.

4.- ¿Qué otros factores considera que pueden incidir en el resultado de la evaluación?

Estar preparado con un buen curriculum como varias maestrías, doctorados y certificaciones. Ser un maestro actualizado. Poner retos a los alumnos y promover su participación.

5.- ¿Qué recomendaría a un colega para mejorar su práctica docente?

Ser dinámicos porque las generaciones cambian. No solo basarse en mostrar diapositivas o un Excel. Vincular a los alumnos con el entorno en proyectos finales o parciales. Realizar viajes de prácticas a las empresas y cuestionarlos sobre mejoras que se pudieran realizar. Fomentar el trabajo colaborativo con lo alumnos y cambiar roles en los equipos.

6.- ¿Considera que el tipo de materia influye en la evaluación?

$\mathrm{Si}$, hay materias que se prestan para ser más interactivas o atractivas. Las materias terminales o las de las de especialidad de su carrera les gustan más. Y los profesores reciben mejores calificaciones. Las materias de tronco común son menos bien evaluadas.

\section{7.- Otras aportaciones.}

No pienso que el número de reprobados influye en la calificación hacia el maestro. Si el alumno califica mal al profesor, es porque verdaderamente el profesor no ha cumplido. Las generaciones han disminuido sus promedios porque le toma más esfuerzo por más actividades que se les asignan. Las nuevas generaciones son menos proclives al trabajo colaborativo pero aprenden. Porque el factor de que son coevaluados por sus compañeros. El ECOA lo considero adecuado porque va cambiando y a los maestros nos y obliga a trabajar más y mejorar los cursos. Creo que la evolución del ECOA será constante.

Respecto a los alumnos, ahora son más exigentes porque salen de intercambio a otras escuelas y a otros países y traen nuevos puntos de comparación. Por último, la práctica de evaluación de los profesores por parte de su jefe es buena porque obliga a los profesores a considerar importante los servicios hacia la institución.

A la profesora le enriquece dar clases en grupos de Honors y multiculturales porque demandan más del maestro y aprende de sus alumnos. Son grupos retadores. 


\section{Apéndice B}

Guía para la observación de características personales y de práctica docente de profesores universitarios

Propósito de la observación:

Percibir los elementos de conducta y acción en profesores y alumnos, relacionados con características personales y de práctica docente de profesores universitarios

Identificar elementos de relación de estas prácticas y características con la evaluación del desempeño de los profesores

\section{Características que deben incluirse en la observación:}

El contexto, ¿cómo es el medio ambiente físico donde se desarrolla la interacción entre profesor y alumnos?, ¿cuáles son los elementos que componen el contexto?, ¿qué tipos de comportamientos promueve el contexto?

Los participantes. Describir a los participantes, las escenas, cuántas personas hay y qué roles asumen, ¿qué los hace estar juntos? Interpretar sus emociones y estados de ánimo.

Actividades e interacción, ¿qué sucede?, definir una secuencia de actividades, ¿cuál es la interacción entre las personas y las actividades?

Frecuencia y duración de la situación observada, ¿cuándo inicio la situación?, ¿cuánto tiempo duró?, ¿es una situación que se repite o es única?

Factores sutiles como actividades que emergen, que son informales y no planeadas, significado simbólico y connotativo de las palabras, comunicación no verbal y situaciones inusuales. 


\section{Apéndice C}

Cuestionario aplicado a alumnos del nivel profesional en el Tecnológico de Monterrey, Campus Monterrey del Departamento Académico de Contabilidad, para evaluación del desempeño docente de sus professores

\begin{tabular}{|c|c|}
\hline PREGUNTA & DESCRIPCIÓN \\
\hline 1 & Inicia las sesiones puntualmente (solo cursos presenciales). \\
\hline 2 & Mantiene el orden y la disciplina en el grupo. \\
\hline 3 & Evalúa todas las actividades que señala la agenda del curso. \\
\hline 4 & $\begin{array}{l}\text { Registra las calificaciones de las actividades evaluables de forma puntual en } \\
\text { la plataforma tecnológica. }\end{array}$ \\
\hline 5 & $\begin{array}{l}\text { Provee retroalimentación clara y oportuna de acuerdo a los tiempos de } \\
\text { respuesta establecidos. }\end{array}$ \\
\hline 6 & $\begin{array}{l}\text { Fomenta una comunicación efectiva, se mantiene abierto (a) al diálogo y crea } \\
\text { un ambiente de confianza y respeto. }\end{array}$ \\
\hline 7 & Comunica y cubre todos los objetivos del curso. \\
\hline 8 & $\begin{array}{l}\text { Fomenta una comunicación efectiva, se mantiene abierto (a) al diálogo y crea } \\
\text { un ambiente de confianza y respeto. }\end{array}$ \\
\hline 9 & $\begin{array}{l}\text { Promueve y asesora a los alumnos, tanto en su trabajo individual como en el } \\
\text { trabajo en equipo. }\end{array}$ \\
\hline 10 & Utiliza material didáctico complementario para apoyar el tema de la clase. \\
\hline 11 & Manifiesta dominio del curso que está impartiendo. \\
\hline 12 & La labor de mi profesor ha sido excelente. \\
\hline
\end{tabular}

Fuente: Vicerrectoría Académica del Tecnológico de Monterrey

\section{Breve CV de los autores}

\section{José Osvaldo Galván Salinas}

Ingeniero en electrónica y comunicaciones, en ITESM Campus Querétaro. Maestría en Ciencias en Ingeniería Industrial con especialidad en Calidad, en el Tecnológico de Celaya. Maestría en Educación con acentuación en desarrollo cognitivo, ITESM modalidad en línea. Consultor en sistemas de calidad y desarrollo profesional en diversas organizaciones de la industria automotriz y ferroviaria. Docente en clases de maestría en Sistemas de Calidad. ORCID ID: 0000-0002-8493-1093. Email: a00291353@itesm.mx

\section{Gabriela María Farías Martínez}

Contador Público, por el Tecnológico de Monterrey, Campus Monterrey, 1991. MBA, Tecnológico de Monterrey, EGADE Business School, Monterrey, 1994. Maestría en Mercadotecnia, Tecnológico de Monterrey, EGADE Business School, Monterrey, 2001. Doctorado en Innovación Educativa, Tecnológico de Monterrey, Campus Tec Virtual, Monterrey, 2006. Especialidad en Ciencias de la Contabilidad, University of Texas at Dallas, U.S.A., 2013. Actualmente es Directora del Departamento Académico de Contabilidad y Finanzas del Tecnológico de Monterrey, Campus Monterrey, México. ORCID ID: 0000-0001-6359-6014. Email: gabriela.farias@itesm.mx 